\title{
Correlation of Adventitial Vasa Vasorum with Intracranial Atherosclerosis: A Postmortem Study
}

\author{
Lu Zheng, ${ }^{a}$ Wen Jie Yang, ${ }^{\mathrm{b}}$ Chun Bo Niu, ${ }^{\mathrm{c}}$ Hai Lu Zhao, ${ }^{\mathrm{d}}$ Ka Sing Wong, ${ }^{\mathrm{a}}$ Thomas Wai Hong Leung, ${ }^{\mathrm{a}}$ \\ Xiang Yan Chen ${ }^{\mathrm{e}}$ \\ a Department of Medicine and Therapeutics, Prince of Wales Hospital, The Chinese University of Hong Kong, Hong Kong \\ ${ }^{\circ}$ The Russell H. Morgan Department of Radiology and Radiological Sciences, The Johns Hopkins Hospital, Baltimore, MD, USA \\ 'Department of Pathology, China-Japan Union Hospital of Jilin University, Jilin, China \\ ${ }^{d}$ Center for Diabetic Systems Medicine, Guangxi Key Laboratory of Excellence, Guilin Medical University, Guilin, China \\ eDepartment of Health Technology and Informatics, The Hong Kong Polytechnic University, Hong Kong
}

Background and Purpose Vasa vasorum (W) have been believed to be rare or non-existent in small-caliber intracranial arteries. In a series of human cerebral artery specimens, we identified and examined the distribution of $\mathrm{W}$ in association with co-existing intracranial atherosclerosis.

Methods We obtained cerebral artery specimens from 32 consecutive autopsies of subjects aged 45 years or above. We scrutinized middle cerebral artery (MCA), vertebral artery (VA), and basilar artery (BA) for the presence of adventitial $\mathrm{W}$. We described the distribution of $\mathrm{W}$, and the characteristics of co-existing atherosclerotic lesions.

Results Among 157 intracranial arteries, adventitial W were present in 74 of the 157 specimens (47\%), involving MCA $(n=13,18 \%), B A(n=14,19 \%)$, and VA $(n=47,64 \%)$. Although qualitatively these 74 adventitial $W$ distributed similarly in arteries with or without atherosclerotic lesions (disease-free arteries $n=4 / 8$; arteries of pre-atherosclerosis $n=17 / 42$; and arteries of progressive atherosclerosis $n=53 / 107$ ), the presence of adventitial $W$ in intracranial VA was associated with a heavier plaque load $\left(1.72 \pm 1.66 \mathrm{~mm}^{2}\right.$ vs. $\left.0.40 \pm 0.32 \mathrm{~mm}^{2}, P<0.001\right)$, severer luminal stenosis $(25 \% \pm 21 \%$ vs. $12 \% \pm 9 \%, P=0.002)$, higher rate of concentric lesions (79\% vs. $36 \%, P=0.002)$, and denser intraplaque calcification ( $44 \%$ vs. $0 \%, P=0.003$ ). Histologically, intracranial VA with W had a larger diameter $(3.40 \pm 0.79 \mathrm{~mm}$ vs. $2.34 \pm 0.58 \mathrm{~mm}, P<0.001)$, thicker arterial wall $(0.31 \pm 0.13 \mathrm{~mm}$ vs. $0.23 \pm 0.06 \mathrm{~mm}, P=0.002)$, and a larger intima-media $(0.19 \pm 0.09 \mathrm{~mm}$ vs. $0.13 \pm 0.04 \mathrm{~mm}$, $P=0.003$ ) than VA without W.

Conclusions Our study demonstrated the distribution of adventitial $\mathrm{W}$ within brain vasculature and association between vertebral $\mathrm{W}$ and progressive atherosclerotic lesions with a heavier plaque load and denser intraplaque calcification.
Correspondence: Xiang Yan Chen Department of Health Technology and Informatics, The Hong Kong Polytechnic University, Hung Hom, Kowloon, Hong Kong

Tel: +852-34008891

Fax: $+852-23624365$

E-mail: fiona.chen@polyu.edu.hk

Co-correspondence: Thomas Wai Hong Leung

Department of Medicine and

Therapeutics, Prince of Wales Hospital, The Chinese University of Hong Kong,

Shatin, Hong Kong

Tel: +852-26352130

Fax: +852-26493761

E-mail:drtleung@cuhk.edu.hk

Received: April 30, 2018

Revised: August 6, 2018

Accepted: August 7, 2018

Keywords: Atherosclerosis; Angiogenesis; Vasa vasorum 


\section{Introduction}

Atherosclerosis is a chronic progressive disease characterized by lipid accumulation in the vascular wall and fibrous cap formation. ${ }^{1,2}$ Intracranial atherosclerosis is a major cause of stroke and may account for $30 \%$ to $50 \%$ of ischemic events in Asians..$^{3-5}$ As a critical contributor to the development of atherosclerosis, inflammation has been shown to progress inward from adventitia to intima. $2,6,7$

Histologically, adventitia consists of connective tissue, fibroblasts, and vasa vasorum (W). ${ }^{1} \mathrm{~W}$ constitute a network of microvasculature and play a nutritive role with drainage capacity in the arterial vessel walls. ${ }^{8}$ Studies have shown that $\mathrm{W}$ may function as a conduit for transporting inflammatory mediators ${ }_{1}^{9-11}$ and therefore, may play a passive role in the pathogenesis of atherosclerosis, aneurysm, vasculitis, and graft vascular disease. ${ }^{11,12}$ Adventitial $\mathrm{W}$ in coronary and carotid arteries may contribute to the vulnerability of atherosclerotic plaques. ${ }^{10}$ However, the ability of cerebral arteries to receive nourishment from surrounding cerebrospinal fluid may help explain the relative absence of intracranial $W$ from early life. ${ }^{13}$

Previous autopsy studies reported intracranial $\mathrm{W}$ by immunohistochemical examination. ${ }^{14,15}$ Nevertheless, compared with the extracranial $W$, knowledge on the histopathology of intracranial $\mathrm{W}$ is scarce, partly owing to the relative inaccessibility of intracranial arteries. Understanding adventitial W development in cerebral arteries and its relationship to atherosclerosis may shed light on mechanisms of ischemic stroke due to presumed intracranial atherosclerosis. Based on a series of human cerebral artery specimens in our biobank, ${ }^{16-19}$ we investigated the distribution of adventitial $\mathrm{W}$ in intracranial large arteries and studied its potential association with co-existing atherosclerosis.

\section{Methods}

\section{Participants}

We selected 32 Chinese autopsies from December 2003 to
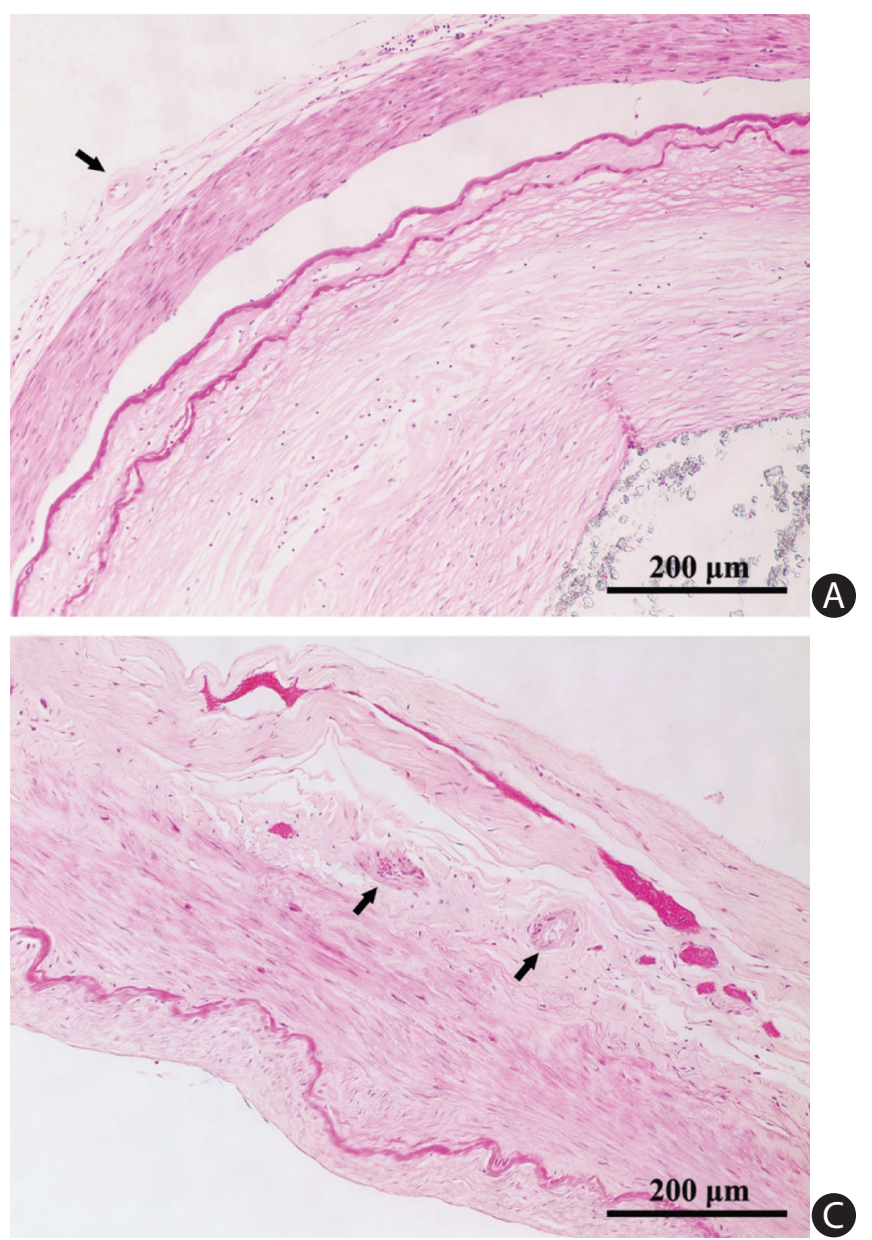
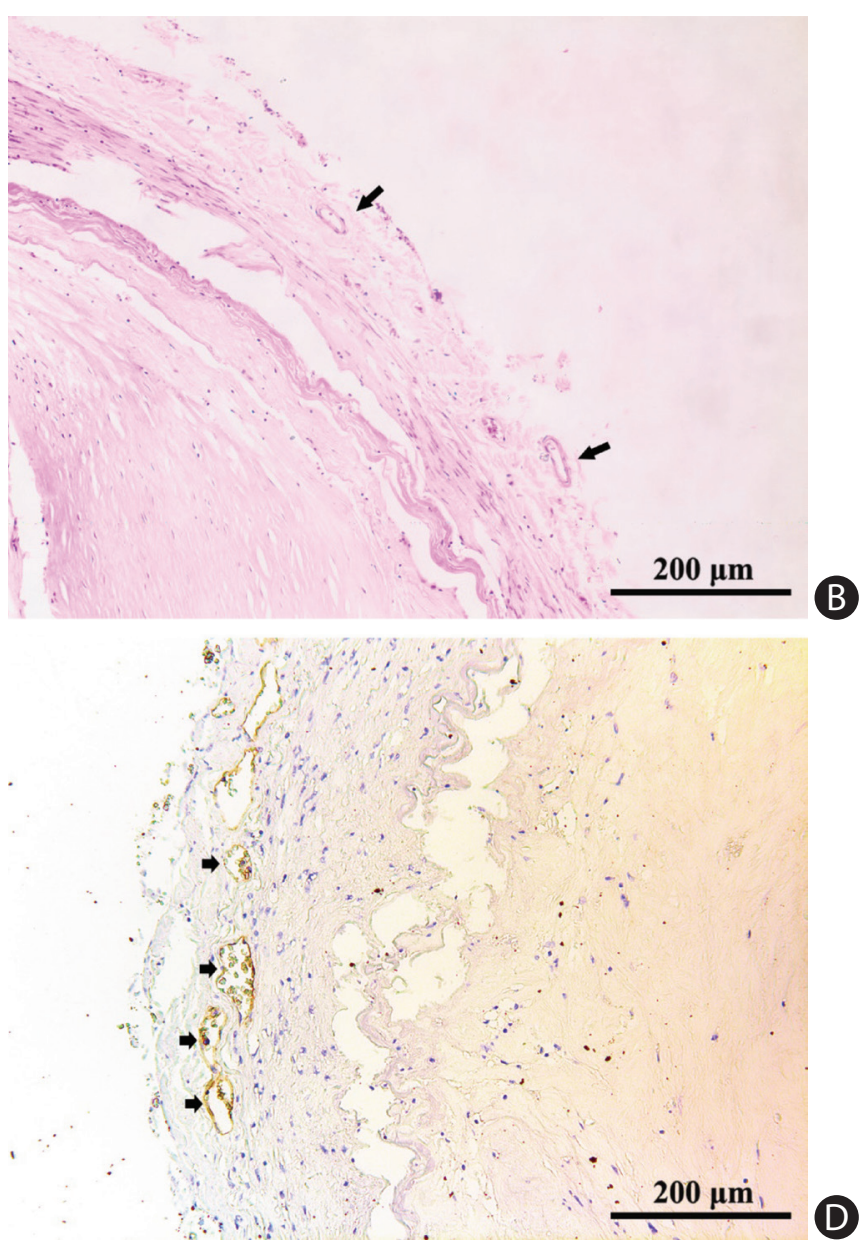

Figure 1. Vasa vasorum in adventitia of cerebral arteries (arrows). (A) Middle cerebral artery (H\&E stain). (B) Basilar artery (H\&E stain). (C) Vertebral artery (H\&E stain). (D) Immunochemical staining for von Willebrand factor revealed multiple vasa vasorum (short arrows). 
June 2005 in the Prince of Wales Hospital, Hong Kong. ${ }^{16}$ Clinical Research Ethics Committee of the Chinese University of Hong Kong approved the study. Pathologists who performed the autopsy were blinded to the study purpose. We obtained subject demographics and clinical data from hospital electronic records. The causes of death were cardiovascular disease ( $n=13,41 \%$, e.g., coronary artery disease, hypertensive heart disease, ischemic stroke, or brain hemorrhage); infection or sepsis $(n=3,9 \%)$; other natural causes ( $n=13,41 \%$, e.g., hepatitis); or unnatural causes ( $n=3,9 \%$, e.g., suicides or accidents).

\section{Histopathology}

We sampled 157 intracranial arteries from 32 autopsy cases, including $M 1$ segments of bilateral middle cerebral arteries ( $M C A, n=64)$, basilar artery $(B A, n=32)$, and $V 4$ segments of bilateral vertebral arteries (VA, $n=61$; three individuals had a single VA). We obtained and labeled each $4 \mathrm{~mm}$ cross-sectional cut of cerebral arteries from formalin-fixed brains. Each segment was decalcified overnight in 10\% formic acid, followed by perfusion fixation in fresh 30\% formaldehyde. After embedding in paraffin, five-micron-thick cuts were obtained from each arterial block for staining (one section per staining) with hematoxylin and eosin (H\&E) for structural evaluation. We used Victoria Blue staining to mark the internal elastic lamina (IEL) for morphological measurements. For immunohistochemistry, we used Abcam (Cambridge, UK) antigen retrieval solution to retrieve antigen. The sections were then incubated with 3\% hydrogen peroxide for 15 minutes and bovine serum albumin for 1 hour and stained with the following primary antibodies: von Willebrand factor (vWF) (1:200, Agilent Dako, Santa Clara, CA, USA) and CD68 (1:200, Sigma, St. Louis, MO, USA).

We photographed the slides with a Leica DC 200 digital microscope (Leica, Witzlar, Germany). Two pathologists (H.L.Z. and C.B.N.) blinded to clinical data examined all histological sections to grade atherosclerotic lesions and to record the plaque features. Another two investigators (L.Z. and W.J.Y.) independently located and described the distribution of W: adventitial W were identified by H\&E staining (Figure $1 \mathrm{~A}-\mathrm{C}$ and Supple-
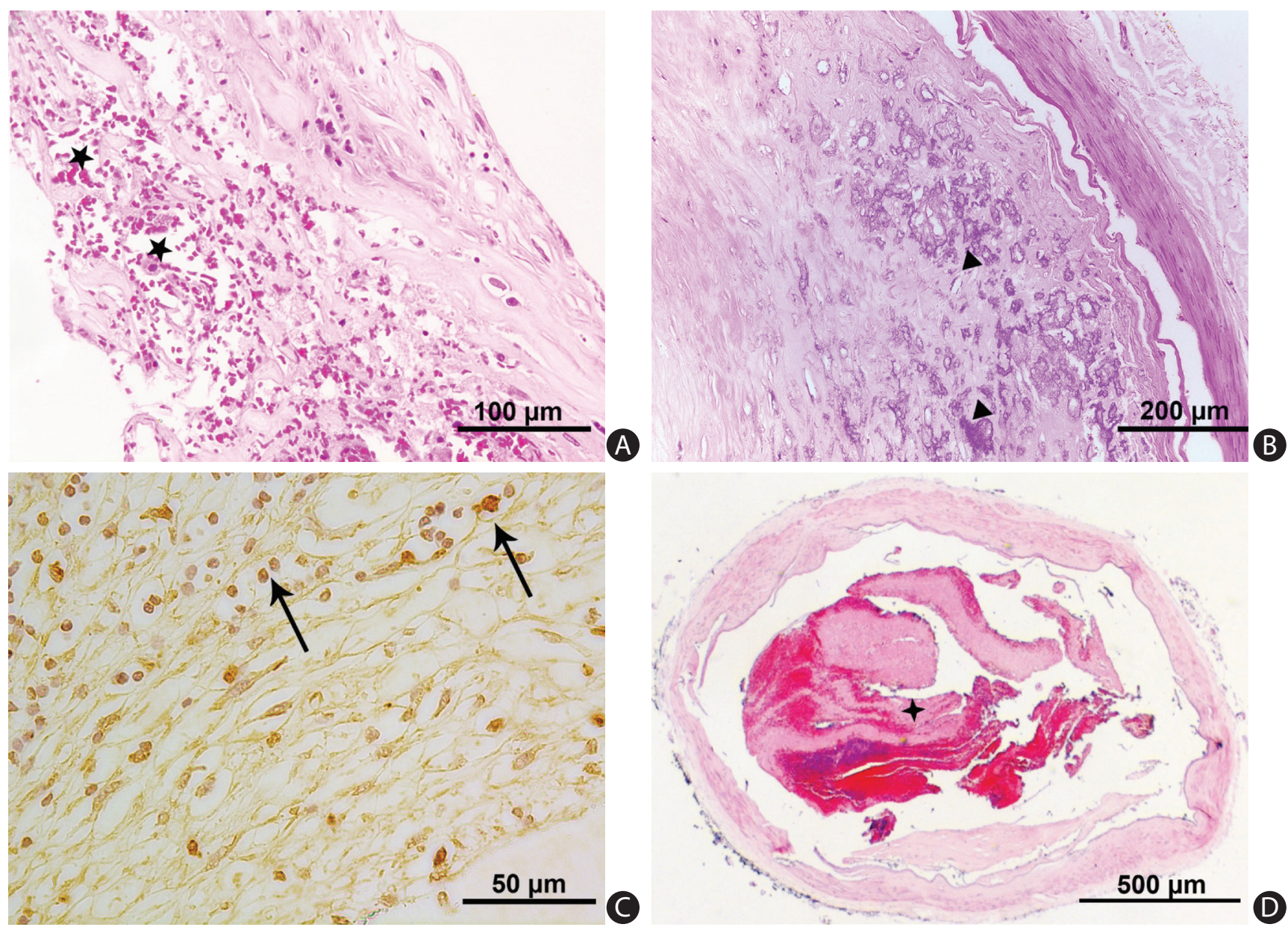

Figure 2. Plaque composition in intracranial arteries. (A) Intraplaque hemorrhage (asterisks) (H\&E stain). (B) Calcification (arrowheads) (H\&E stain). (C) Macrophage infiltration (long arrows) (CD68 antibodies staining). (D) Lumen thrombi (asterisk) (H\&E stain). 
mentary Figure 1), as well as immunostaining that outlined the diffuse perivascular deposits of vWF surrounding a microvessel (Figure 1D). Based on the revised American Heart Association (AHA) criteria, ${ }^{20}$ we classified the arteries into three groups: (1) disease-free, with normal intima; (2) pre-atherosclerotic lesions showing intimal thickening or intimal xanthoma; and (3) progressive atherosclerotic lesions showing pathologic intimal thickening, fibrous cap atheroma or fibrocalcific plaque. Concentric lesions were plaques involving the entire circumference of the ILE, whereas eccentric plaques had intervening diseasefree wall. ${ }^{21}$ We recorded the characteristics of a complicated plaque, if any, including intraplaque hemorrhage (Figure 2A), calcification (Figure 2B), macrophage infiltration (Figure 2C), and thrombus (Figure 2D).

\section{Image analysis}

We used Image-Pro Plus software to assess arterial structure. Based on the method described by Gutierrez et al., ${ }^{22}$ we measured arterial diameter, wall thickness, intima-media thickness, adventitia thickness, area of atherosclerotic plaque (plaque load), and percentage of luminal narrowing (area stenosis). We traced the border of external adventitia, media, IEL and plaque manually and calculated the length

Table 1. Clinical characteristics of 32 autopsy cases

\begin{tabular}{lc}
\hline Characteristic & Total \\
\hline Age (yr) & $71(45-97)$ \\
Male sex & $23(72)$ \\
Smoker & $9(28)$ \\
Hypertension & $9(28)$ \\
Diabetes & $6(19)$ \\
Ischemic heart disease & $9(28)$ \\
Ischemic stroke & $14(44)$ \\
Hemorrhagic stroke & $2(6)$ \\
\hline
\end{tabular}

Values are presented as median (interquartile range) or number (\%). and area with software. After outlining the perimeters of different layers as $P_{\text {outer, }} P_{\text {media, }}$ and $P_{\text {IELL }}$ we calculated $R_{\text {artery, }}$ $R_{\text {media, }}$ and $R_{\text {IEL }}$ by radius = perimeter $/ 2 \pi$. The thicknesses (Th) of adventitia and intima-media were calculated as folIows: $T h_{\text {adventitia }}=R_{\text {artery }}-R_{\text {media, }} T h_{\text {intima-media }}=R_{\text {media }}-R_{\text {IEL. }}$ The wall thickness was the sum of $T h_{\text {adventitia }}$ and $T h_{\text {intima-media. We }}$ derived the luminal area by $A_{I E L}=P_{I E L}{ }^{2} / 4 \pi$, and the area stenosis by (plaque load / $\left.A_{\text {IEL }}\right) \times 100$.

\section{Statistical analysis}

We analyzed data using SPSS version 20.0 software package (IBM Co., Armonk, NY, USA). For comparisons among different cerebral arteries for the 32 subjects, we used mean values of the MCAs and VAs for a given autopsy case and comparisons were made using chi-square test for categorical data and paired t-test for continuous variables. Comparisons between arteries with and without adventitial W were made by independent sample t-test for continuous variables and chisquare test for categorical variables. Results are presented as mean \pm SD. We considered $P<0.05$ as statistically significant. For multiple testing, a significance level was considered as $0.016(P=0.05 / 3)$ by Bonferroni correction.

\section{Results}

\section{Sample description}

We selected all 32 Chinese adult autopsies aged 45 years or above from December 2003 to June 2005. Median age was 71 years (range, 45 to 97). Twenty-three (72\%) were male. For cardiovascular risk factors, smoking was found in nine cases $(28 \%)$, hypertension in nine $(28 \%)$, and diabetes mellitus in six (19\%). For the history of clinical cardiovascular events, nine had ischemic heart disease (28\%), 14 had ischemic stroke (44\%), and two had hemorrhagic stroke (6\%). Table 1 summarizes the demographics and risk factors of the included cases.

Table 2. Histologic features of different intracranial arteries

\begin{tabular}{lcccccc}
\hline Variable & Middle cerebral artery & Basilar artery & Vertebral artery & $P^{*}$ & $P^{*}$ & $P^{\S}$ \\
\hline Arterial diameter $(\mathrm{mm})$ & $3.08 \pm 0.57$ & $3.41 \pm 0.66$ & $3.19 \pm 0.62$ & $0.009^{\prime \prime}$ & 0.311 & 0.147 \\
Wall thickness $(\mathrm{mm})$ & $0.22 \pm 0.08$ & $0.26 \pm 0.08$ & $0.29 \pm 0.10$ & 0.017 & $0.001^{\prime \prime}$ & 0.056 \\
Intima-media thickness $(\mathrm{mm})$ & $0.16 \pm 0.07$ & $0.19 \pm 0.07$ & $0.18 \pm 0.07$ & $0.016^{\prime \prime}$ & 0.305 & 0.119 \\
Adventitia thickness $(\mathrm{mm})$ & $0.06 \pm 0.03$ & $0.06 \pm 0.02$ & $0.11 \pm 0.05$ & 0.217 & $0.001^{\prime \prime}$ & $0.001^{\prime \prime}$ \\
Plaque load $\left(\mathrm{mm}^{2}\right)$ & $1.94 \pm 1.75$ & $1.86 \pm 2.06$ & $1.42 \pm 1.14$ & 0.768 & 0.070 & 0.118 \\
Area stenosis $(\%)$ & $31 \pm 20$ & $20 \pm 19$ & $22 \pm 15$ & $0.001^{\prime \prime}$ & $0.006^{\prime \prime}$ & 0.571 \\
\hline
\end{tabular}

Values are presented as mean \pm SD.

*In each subject, the means for middle cerebral and vertebral arteries were utilized in the analysis; Comparisons made between: ${ }^{+}$middle cerebral artery and basilar artery; ${ }^{*}$ middle cerebral artery and vertebral artery; and ${ }^{5}$ vertebral artery and basilar artery; "Bonferroni corrected significance level $P<0.016$. 


\section{Anatomy and distribution of adventitial W}

We scrutinized 157 intracranial arteries: $64 \mathrm{MCAs}, 32 \mathrm{BAs}$, and 61 VAs. Table 2 shows comparison of histologic features among different intracranial arteries. MCA had a thinner arterial wall than VA $(0.22 \pm 0.08 \mathrm{~mm}$ vs. $0.29 \pm 0.10 \mathrm{~mm}, P<0.016)$. VA had a significantly thicker adventitia $(0.11 \pm 0.05 \mathrm{~mm}, P<0.016)$ than MCA or BA. BA had a larger artery diameter $(3.41 \pm 0.66 \mathrm{~mm}$ vs. $3.08 \pm 0.57 \mathrm{~mm}, P<0.016)$ and a thicker intima-media $(0.19 \pm 0.07 \mathrm{~mm}$ vs. $0.16 \pm 0.07 \mathrm{~mm}, P<0.016)$ than MCA. The area stenosis (\%) was greatest in MCA compared with BA or VA $(31 \% \pm 20 \%$ vs. $20 \% \pm 19 \%$ or $31 \% \pm 20 \%$ vs. $22 \% \pm 15 \%$; $P<0.01$, respectively). Adventitial $W$ were most prevalent in $V A$ (47/61) compared with MCA (13/64) or BA (14/32) (77\% vs. $20 \%$ or $77 \%$ vs. $44 \%$; $P<0.016$, respectively).

\section{Association between $\mathrm{W}$ and phenotypes of atherosclerosis}

Based on the revised AHA criteria, eight arteries (5\%) were disease-free with normal intima, 42 (27\%) had pre-atherosclerotic lesions, and 107 (68\%) had progressive atherosclerotic lesions. Among all 157 arterial distributed segments, we identified adventitial W in 74 specimens (47\%): 13 MCAs (18\%), 14 BAs (19\%), and 47 VAs (64\%). In terms of co-existing atherosclerosis, these 74 adventitial $\mathrm{W}$ were present in four disease-free segments (5\%), in 17 segments with pre-atherosclerotic lesions (23\%), and in 53 segments with progressive atherosclerotic lesions (72\%). In MCA and BA, the prevalence of atherosclerotic lesions did not significantly differ between arteries with adventitial $\mathrm{W}$ and those without. However, in VA, arteries with adventitial $\mathrm{W}$ were likely to have more co-existing progressive atherosclerotic lesions compared to arteries without adventitial W (68\% vs. $29 \%, P<0.05$ ) (Table 3 ).

Table 3. Adventitial vasa vasorum and stages of atherosclerosis

\begin{tabular}{lccc}
\hline Adventitial vasa vasorum & Disease free & Pre-atherosclerotic lesions & Progressive atherosclerotic lesions \\
\hline Middle cerebral artery & & & $P$ \\
Present $(n=13)$ & $0(0)$ & $2(15)$ & $11(85)$ \\
Absent $(n=51)$ & $3(12)$ & $8(16)$ & $40(78)$ \\
Basilar artery & & $2(14)$ & $10(71)$ \\
Present $(n=14)$ & $2(14)$ & $7(39)$ & $10(56)$ \\
Absent $(n=18)$ & $1(6)$ & & $32(68)$ \\
Vertebral artery & & $13(28)$ & $4(29)$ \\
Present $(n=47)$ & $2(4)$ & $10(71)$ & $107(68)$ \\
Absent $(n=14)$ & $0(0)$ & $42(27)$ & 0.543 \\
Total $(n=157)$ & $8(5)$ & & $0.016^{*}$ \\
\hline
\end{tabular}

Values are presented as number (\%).

${ }^{*} P<0.05$.

Table 4. Adventitial vasa vasorum and atherosclerosis in vertebral artery

\begin{tabular}{lcccc}
\hline Variable & $\begin{array}{c}\text { All vertebral arteries } \\
(\mathrm{n}=61)\end{array}$ & $\begin{array}{c}\text { Arteries with adventitial } \\
\text { vasa vasorum }(\mathrm{n}=47)\end{array}$ & $\begin{array}{c}\text { Arteries without adventitial } \\
\text { vasa vasorum }(\mathrm{n}=14)\end{array}$ & $P$ \\
\hline Arterial diameter $(\mathrm{mm})$ & $3.19 \pm 0.62$ & $3.40 \pm 0.79$ & $2.34 \pm 0.58$ & $0.001^{*}$ \\
Wall thickness $(\mathrm{mm})$ & $0.29 \pm 0.10$ & $0.31 \pm 0.13$ & $0.23 \pm 0.06$ & $0.002^{*}$ \\
Intima-media thickness $(\mathrm{mm})$ & $0.18 \pm 0.07$ & $0.19 \pm 0.09$ & $0.13 \pm 0.04$ & $0.003^{*}$ \\
Adventitia thickness $(\mathrm{mm})$ & $0.11 \pm 0.05$ & $0.12 \pm 0.07$ & $0.09 \pm 0.04$ & 0.175 \\
Plaque load $\left(\mathrm{mm}^{2}\right)$ & $1.42 \pm 1.14$ & $1.72 \pm 1.66$ & $0.40 \pm 0.32$ & $0.001^{*}$ \\
Area stenosis $(\%)$ & $22 \pm 15$ & $25 \pm 21$ & $12 \pm 9$ & $0.002^{*}$ \\
Concentric distribution & $42(69)$ & $37(79)$ & $5(36)$ & $0.002^{*}$ \\
Intraplaque hemorrhage & $7(12)$ & $7(15)$ & $0(0)$ & $1(7)$ \\
Thrombus & $6(10)$ & $5(11)$ & $3(21)$ & 0.125 \\
Macrophages & $24(39)$ & $21(45)$ & 0.700 & 0.118 \\
Calcification & $20(33)$ & $20(43)$ & $0.003^{*}$ & \\
\hline
\end{tabular}

Values are presented as mean \pm SD or number (\%).

${ }^{*} P<0.05$. 


\section{Association between adventitial $\mathrm{W}$ and atherosclerotic surrogates in VA}

Table 4 compares the anatomy and atherosclerotic features in VA with and without $W$. The VA with adventitial $W$ had a larger diameter $(3.40 \pm 0.79 \mathrm{~mm}$ vs. $2.34 \pm 0.58 \mathrm{~mm}, P<0.001)$, a thicker arterial wall $(0.31 \pm 0.13 \mathrm{~mm}$ vs. $0.23 \pm 0.06 \mathrm{~mm}$, $P=0.002)$, and a thicker intima-media $(0.19 \pm 0.09 \mathrm{~mm}$ vs. $0.13 \pm 0.04 \mathrm{~mm}, P=0.003)$ than VA without W. The adventitial $W$ in $V 4$ segments was associated with a heavier plaque load $\left(1.72 \pm 1.66 \mathrm{~mm}^{2}\right.$ vs. $\left.0.40 \pm 0.32 \mathrm{~mm}^{2}, P<0.001\right)$, severer area stenosis ( $25 \% \pm 21 \%$ vs. $12 \% \pm 9 \%, P=0.002)$, higher rate of concentric lesions ( $79 \%$ vs. $36 \%, P=0.002$ ), and denser intraplaque calcification ( $43 \%$ vs. $0 \%, P=0.003$ ).

\section{Discussion}

In this autopsy study, we found adventitial W in nearly half $(74 / 157,47 \%)$ of all intracranial arterial segments, predominantly in V4 segments of VA (64\%), followed by BA (19\%) and MCA (18\%). Overall, adventitial W were present in disease-free arteries $(4 / 8,50 \%)$ as well as in arteries with pre-atherosclerotic $(17 / 42,40 \%)$ or progressive atherosclerotic lesions (53/107, 50\%). In VA, adventitial W were associated with concentric steno-occlusive atherosclerotic lesions and denser intraplaque calcification.

W are composed of artery, capillary, and vein that deliver oxygen and nutrition to and eliminate metabolic wastes from the vessel wall. ${ }^{23,24}$ In contrast to the high prevalence of $W$ in extracranial arteries, the existence of $W$ in human intracranial arteries had been a debate due to paucity of cerebral specimens. In 1980s, although Zervas et al..$^{25}$ and Clower et al. ${ }^{26}$ reported no $\mathrm{W}$ in cerebral arteries in animal studies, $\mathrm{W}$ were revealed in adventitia of internal carotid artery (ICA), anterior cerebral artery and MCA of five humans, suggesting that W might be species-specific. ${ }^{14}$ In a larger autopsy series of 15 cases aged 5 days to 86 years, Aydin ${ }^{15}$ subsequently revealed the presence of $W$ in proximal intracranial segments of ICA and VA but not in BA or MCA. However, in the current study of a larger sample size, we noted that $W$ were frequently found in the vertebral-basilar circulation (80\% in V4 segments of VA and $44 \%$ of BA), but much less in MCA (only 20\%).

In our study, the adventitia of VA was significantly thicker than that of MCA and BA, and thus, the higher prevalence of $W$ in VA might correspond to a higher metabolic demand in a thicker vessel wall where diffusion from parent arterial lumen or CSF for nutrition or oxygen might be insufficient. A previous study reported that the extent and distribution of $W$ depended on the medial thickness. ${ }^{27}$ In fact, arterial wall and intima-media layers in VA with adventitial W were found to be thicker than those without. Our findings along with literature ${ }^{14,15}$ suggest that adventitial $\mathrm{W}$ might exist more frequently in the proximal parts of the intracranial arteries such as VA than in arteries located more distally. As a natural extension of extracranial arteries, VA is likely a transition zone that possesses certain features different from the true intracranial arteries such as MCA and BA.

Previous studies suggested that $\mathrm{W}$ were extremely rare in cerebral arteries and might develop only in pathological conditions. ${ }^{28}$ Our study findings do not substantiate this theory. On the contrary, adventitial $W$ were present in four disease-free arteries from two autopsies devoid of any vasculopathy. Nevertheless, while this finding might support the physiologic existence of $W$ in cerebral arteries, it remains unclear whether $W$ could trigger and exacerbate the development and progression of atherosclerosis.

In 1984, Barger et al..$^{29}$ hypothesized that $W$ could be involved in the process of atherosclerosis. Studies in both human autopsy $^{30}$ and animal models ${ }^{31}$ found higher density of $W$ in unstable atherosclerotic lesions. In a coronary artery disease model, $\mathrm{W}$ may trigger the initial stage of atherosclerosis..$^{32}$ In our study, a higher prevalence of progressive atherosclerotic lesions in V4 segments, with heavier plaque load and severer luminal stenosis were found in conjunction with adventitial $\mathrm{W}$. We postulate that adventitial neovascular network might act as conduits transporting inflammatory cells and mediators into the plaque, exacerbating the pathologic process of atherosclerosis. ${ }^{723}$ In this current study, we found that cerebral arteries with $\mathrm{W}$ were more likely to have atherosclerotic plaques with more hemorrhage. Immature microvessels could act as sites of inflammatory cell infiltration and intraplaque hemorrhage owing to the weak integrity of such vessels..$^{33,34}$ Studies in coronary artery and carotid showed that intraplaque hemorrhage is not only a common feature in advanced atherosclerotic plaques, ${ }^{35}$ but also plays an important role in plaque destabilization which ultimately may lead to clinical ischemic events..$^{12,36-38}$ In cerebral arteries, our previous findings demonstrated that in addition to the luminal stenosis, plaque components such as lipid and intraplaque hemorrhage may be responsible for the brain infarct. ${ }^{16}$ Therefore, adventitial W may play a nutritive role in the progression of intracranial atherosclerosis and might be considered as a marker of complicated plaques. However, whether adventitial $\mathrm{W}$ in intracranial arteries play a causative role in brain infarction has not been well established yet.

The interpretation of our findings would be limited by the selection bias inherent to a retrospective post-mortem study. 
The heterogeneity of the enrolled autopsy cases could be a major source of confounder and extrapolation to the general population should be treated with caution. Considering the nature of a post-mortem study, we could not provide causal relationship between presence of $\mathrm{W}$ and progression of atherosclerosis within the intracranial vascular beds. Besides, we did not analyze the association between the magnitude of adventitia $\mathrm{W}$ and atherosclerosis in this study. Moreover, although our study has a relatively larger sample size compared with previous studies, the number was still insufficient to allow subgroup analysis to analyze adventitial $W$ in relation to various stages of atherosclerosis and to adequately adjust covariates. Future investigation may shed light on whether and how adventitial $\mathrm{W}$ may impact on the evolution of atherosclerosis.

\section{Conclusions}

In conclusion, our investigation shows high prevalence of adventitial $W$ in intracranial arterial segments of VAs. In VAs, we observed an association between $\mathrm{W}$ and progressive atherosclerotic lesions with a heavier plaque load and denser intraplaque calcification.

\section{Supplementary materials}

Supplementary materials related to this article can be found online at https://doi.org/10.5853/jos.2018.01263.

\section{Disclosure}

The authors have no financial conflicts of interest.

\section{Acknowledgments}

The study was supported by grants from the National Natural Science Foundation of China (Project No. 81371297), the General Research Fund from Research Grants Council of Hong Kong (GRF, Project No. 14112916) and the Health and Medical Research Fund of Hong Kong (HMRF, Project No. 04152586). The funders had no role in study design, data collection and analysis, decision to publish, or preparation of the manuscript.

\section{References}

1. Lusis AJ. Atherosclerosis. Nature 2000;407:233-241.

2. Lu H, Daugherty A. Atherosclerosis: cell biology and lipoproteins. Curr Opin Lipidol 2015;26:152-153.

3. Wong KS, Huang YN, Gao S, Lam WW, Chan YL, Kay R. Intra- cranial stenosis in Chinese patients with acute stroke. Neurology 1998;50:812-813.

4. Wong KS, Li H, Chan YL, Ahuja A, Lam WW, Wong A, et al. Use of transcranial Doppler ultrasound to predict outcome in patients with intracranial large-artery occlusive disease. Stroke 2000:31:2641-2647.

5. Wang Y, Zhao X, Liu L, Soo YO, Pu Y, Pan Y, et al. Prevalence and outcomes of symptomatic intracranial large artery stenoses and occlusions in China: the Chinese Intracranial Atherosclerosis (CICAS) Study. Stroke 2014;45:663-669.

6. Libby P. Inflammation in atherosclerosis. Nature 2002;420: 868-874.

7. Maiellaro $K_{1}$ Taylor WR. The role of the adventitia in vascular inflammation. Cardiovasc Res 2007;75:640-648.

8. Kohnken $R$, Scansen BA, Premanandan C. Vasa vasorum arteriopathy: relationship with systemic arterial hypertension and other vascular lesions in cats. Vet Pathol 2017;54:475483.

9. Moulton KS, Vakili K, Zurakowski D, Soliman M, Butterfield

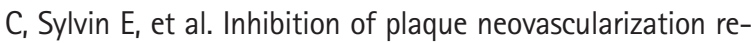
duces macrophage accumulation and progression of advanced atherosclerosis. Proc Natl Acad Sci U S A 2003;100: 4736-4741.

10. Gossl M, Versari D, Mannheim D, Ritman EL, Lerman LO, Lerman A. Increased spatial vasa vasorum density in the proximal LAD in hypercholesterolemia: implications for vulnerable plaque-development. Atherosclerosis 2007;192:246-252.

11. Herrmann J, Lerman LO, Rodriguez-Porcel $M_{1}$ Holmes DR Jr, Richardson DM, Ritman EL, et al. Coronary vasa vasorum neovascularization precedes epicardial endothelial dysfunction in experimental hypercholesterolemia. Cardiovasc Res 2001;51:762-766.

12. Kolodgie FD, Gold HK, Burke AP, Fowler DR, Kruth HS, Weber DK, et al. Intraplaque hemorrhage and progression of coronary atheroma. N Engl J Med 2003;349:2316-2325.

13. Portanova A, Hakakian N, Mikulis DJ, Virmani R, Abdalla WM, Wasserman BA. Intracranial vasa vasorum: insights and implications for imaging. Radiology 2013;267:667-679.

14. Connolly ES Jr, Huang J, Goldman JE, Holtzman RN. Immunohistochemical detection of intracranial vasa vasorum: a human autopsy study. Neurosurgery 1996;38:789-793.

15. Aydin F. Do human intracranial arteries lack vasa vasorum? A comparative immunohistochemical study of intracranial and systemic arteries. Acta Neuropathol 1998;96:22-28.

16. Chen XY, Wong KS, Lam WW, Zhao HL, Ng HK. Middle cerebral artery atherosclerosis: histological comparison between plaques associated with and not associated with infarct in a postmortem study. Cerebrovasc Dis 2008;25:74-80. 
17. Yang WJ, Chen $X Y$, Zhao $H L$, Niu CB, Zhang $B, X u Y$, et al. Postmortem study of validation of low signal on fat-suppressed T1-weighted magnetic resonance imaging as marker of lipid core in middle cerebral artery atherosclerosis. Stroke 2016;47:2299-2304.

18. Yang WJ, Chen $X Y$, Zhao HL, Niu CB, Xu Y, Wong KS, et al. In vitro assessment of histology verified intracranial atherosclerotic disease by $1.5 \mathrm{~T}$ magnetic resonance imaging: concentric or eccentric? Stroke 2016;47:527-530.

19. Yang WJ, Fisher M, Zheng L, Niu CB, Paganini-Hill A, Zhao $H L$, et al. Histological characteristics of intracranial atherosclerosis in a Chinese population: a postmortem study. Front Neurol 2017;8:488.

20. Virmani R, Kolodgie FD, Burke AP, Farb A, Schwartz SM. Lessons from sudden coronary death: a comprehensive morphological classification scheme for atherosclerotic lesions. Arterioscler Thromb Vasc Biol 2000;20:1262-1275.

21. Varnava AM, Mills PG, Davies MJ. Relationship between coronary artery remodeling and plaque vulnerability. Circulation 2002;105:939-943.

22. Gutierrez J, Goldman J, Honig LS, Elkind MS, Morgello S, Marshall RS. Determinants of cerebrovascular remodeling: do large brain arteries accommodate stenosis? Atherosclerosis 2014;235:371-379.

23. Ritman EL, Lerman A. The dynamic vasa vasorum. Cardiovasc Res 2007;75:649-658.

24. Tsikaras DP, Natsis K, Hytiroglou P, Lazos L, Gigis P. Microanatomy of the vasa vasorum of the human thoracic aorta: a study utilizing a polyester resin casting technique. Morphologie 1997;81:21-22.

25. Zervas NT, Liszczak TM, Mayberg MR, Black PM. Cerebrospinal fluid may nourish cerebral vessels through pathways in the adventitia that may be analogous to systemic vasa vasorum. J Neurosurg 1982;56:475-481.

26. Clower BR, Sullivan DM, Smith RR. Intracranial vessels lack vasa vasorum. J Neurosurg 1984;61:44-48.

27. Wolinsky H, Glagov S. Nature of species differences in the medial distribution of aortic vasa vasorum in mammals. Circ Res 1967;20:409-421.

28. Takaba M, Endo S, Kurimoto $M$, Kuwayama N, Nishijima $M$,
Takaku A. Vasa vasorum of the intracranial arteries. Acta Neurochir (Wien) 1998;140:411-416.

29. Barger AC, Beeuwkes R 3rd, Lainey LL, Silverman KJ. Hypothesis: vasa vasorum and neovascularization of human coronary arteries. A possible role in the pathophysiology of atherosclerosis. N Engl J Med 1984;310:175-177.

30. Gossl M, Versari D, Hildebrandt HA, Bajanowski T, Sangiorgi $G$, Erbel $R$, et al. Segmental heterogeneity of vasa vasorum neovascularization in human coronary atherosclerosis. JACC Cardiovasc Imaging 2010;3:32-40.

31. Kwon HM, Sangiorgi G, Ritman EL, McKenna C, Holmes DR $J r$, Schwartz RS, et al. Enhanced coronary vasa vasorum neovascularization in experimental hypercholesterolemia. J Clin Invest 1998;101:1551-1556.

32. Choi BJ, Matsuo Y, Aoki T, Kwon TG, Prasad A, Gulati R, et al. Coronary endothelial dysfunction is associated with inflammation and vasa vasorum proliferation in patients with early atherosclerosis. Arterioscler Thromb Vasc Biol 2014;34:24732477.

33. Carmeliet $P$. Mechanisms of angiogenesis and arteriogenesis. Nat Med 2000;6:389-395.

34. Jain RK. Molecular regulation of vessel maturation. Nat Med 2003;9:685-693.

35. Li X, Vink A, Niessen HW, Kers J, de Boer OJ, Ploegmakers HJ, et al. Total burden of intraplaque hemorrhage in coronary arteries relates to the use of coumarin-type anticoagulants but not platelet aggregation inhibitors. Virchows Arch 2014;465: 723-729.

36. Takaya N, Yuan C, Chu B, Saam T, Polissar NL, Jarvik GP, et al. Presence of intraplaque hemorrhage stimulates progression of carotid atherosclerotic plaques: a high-resolution magnetic resonance imaging study. Circulation 2005;111:27682775.

37. Davies MJ. The pathophysiology of acute coronary syndromes. Heart 2000;83:361-366.

38. Kockx MM, Cromheeke KM, Knaapen MW, Bosmans JM, De Meyer GR, Herman AG, et al. Phagocytosis and macrophage activation associated with hemorrhagic microvessels in human atherosclerosis. Arterioscler Thromb Vasc Biol 2003;23:440446. 
Vol. 20 / No. 3 / September 2018

JoS
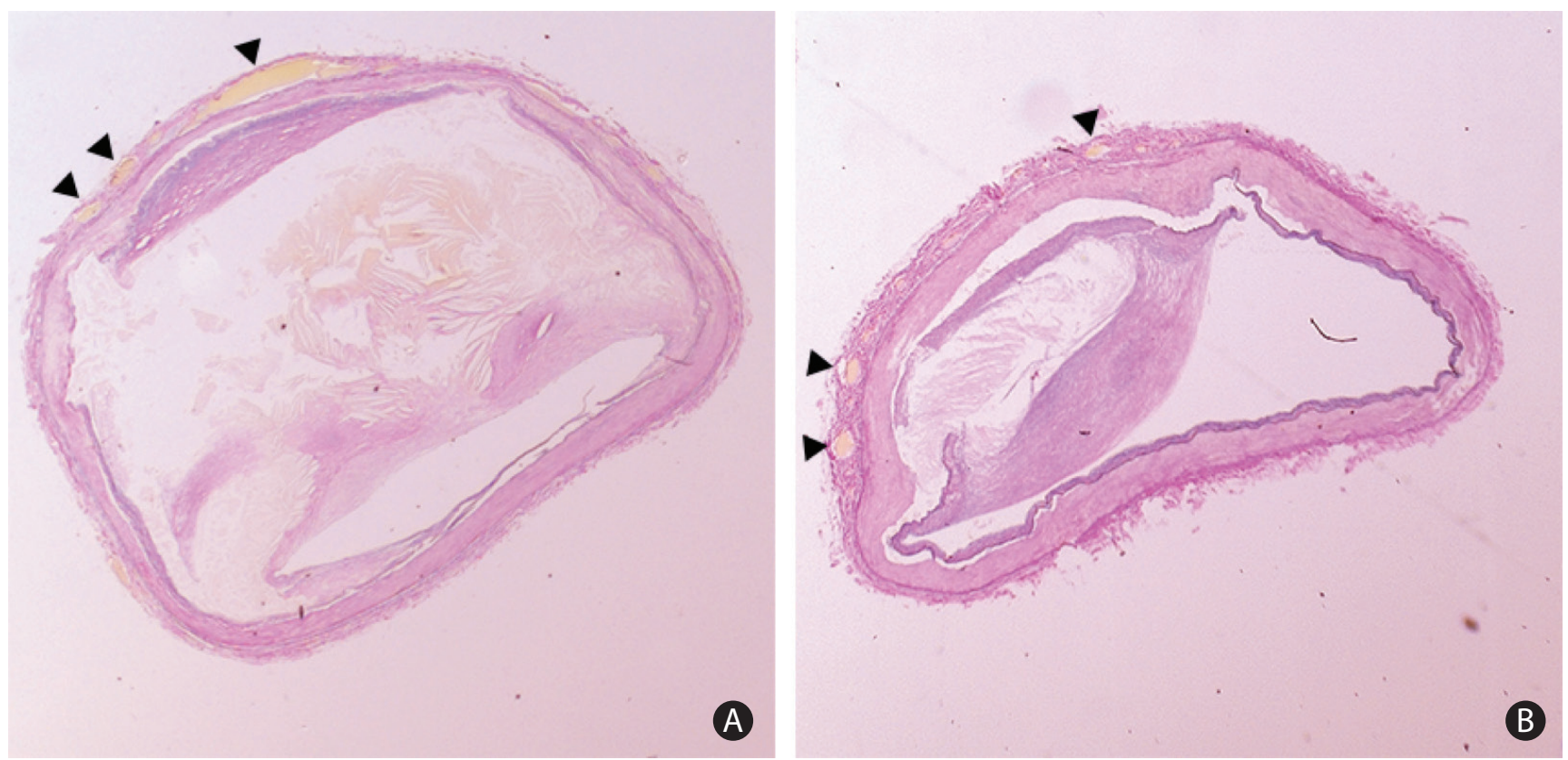

Supplementary Figure 1. (A, B) Vasa vasorum (triangles) of intracranial vertebral artery. These were cross-sections of two vertebral arteries showing vasa vascrum in close anatomic relation with atherosclerotic plaques (H\&E stain, $\times 1.6$ ).

https://doi.org/10.5853/jos.2018.01263

http://j-stroke.org 1 